\title{
EINE NEUE BIBLIOGRAPHISCHE SAMMLUNG ZU EUROPÄISCHEN ORTS-, FLUR- UND GEWÄSSERNAMEN — HINWEISE ZUR BENUTZUNG ${ }^{1}$
}

Schlüsselwörter: Ortsnamen, Flurnamen, Gewässernamen, Europa, Bibliographie

Eine überzeugende Deutung eines geographischen Namens kann nur dann gelingen, wenn umfangreiche Vorarbeiten erfolgt sind. Zum einen ist es notwendig, eine sorgfältige Sammlung der historischen Überlieferung des Namens vorzunehmen, wobei man natürlich auf zuverlässige Editionen der Texte angewiesen ist.

Zum zweiten ist es unerlässlich, nach ähnlichen Namen, nach Vergleichsnamen und nach Namenparallelen zu suchen, sei es für das Grundwort oder die Ableitungsgrundlage - beides ist von großem Nutzen.

Im Grunde gilt dafür ein altes Wort der Onomastik auch noch heute: Erst sammeln, dann deuten. W. Eilers (1982, S. 49) hat das wie folgt ausgedrückt: „Erst sammeln und klassifizieren, dann sprachlich-geschichtlich analysieren! Alle Erfolge, alle Einsichten, die ich auf dem Gebiete der Namenforschung zu verzeichnen habe, sind auf diese Weise gewonnen worden".

Hat man genügend Material für einen Ortsnamen und seine möglichen Parallelen gesammelt, so empfiehlt sich noch ein dritter Weg: die Kartierung des Namens und seiner Parallelen, worunter z. B. Kartierungen des Grundwortes, der Bestimmungswörter und der Namenbildungselemente zu verstehen sind. Th. Frings (1957, S. 9) hat das mit deutlichen Worten unterstrichen: „Den Karten messen wir besondere Bedeutung zu. Ihre plastische Art vermag mehr zu sagen als das Gerede vieler Seiten".

Um diesen Bedingungen — vor allem der letzten — gerecht zu werden, ist lange Sammelarbeit über Jahre und Jahrzehnte hinweg notwendig. Das betrifft alle großen

${ }^{1}$ Wesentliche Teile dieser Abhandlung - außer den Hinweisen auf die Internetdatei - sind von mir schon in der Publikation Udolph (1979) an verschiedenen Stellen publiziert worden. Allerdings sind die Ergebnisse der damaligen Studie, die sich auf die Ausbreitung südslavischer Stämme bezogen haben, von der Fachwelt unberücksichtigt geblieben. Ich halte sie aber für zu wichtig, als dass sie unberücksichtigt bleiben sollten. 
onomastischen Unternehmungen, so z. B. auch die große Sammlung der polnischen Ortsnamen in Kraków (NMPol) oder der altpolnischen Personennamen (SSNO).

$\mathrm{Zu}$ welchen interessanten Ergebnissen die Sammlung und Kartierung slavischer Orts- und Gewässernamen führen können, hat vor Jahrzehnten J. Zaimov (1967, Beilage) anhand einer Verbreitungskarte südslavischer Stämme zeigen können (Karte 1).

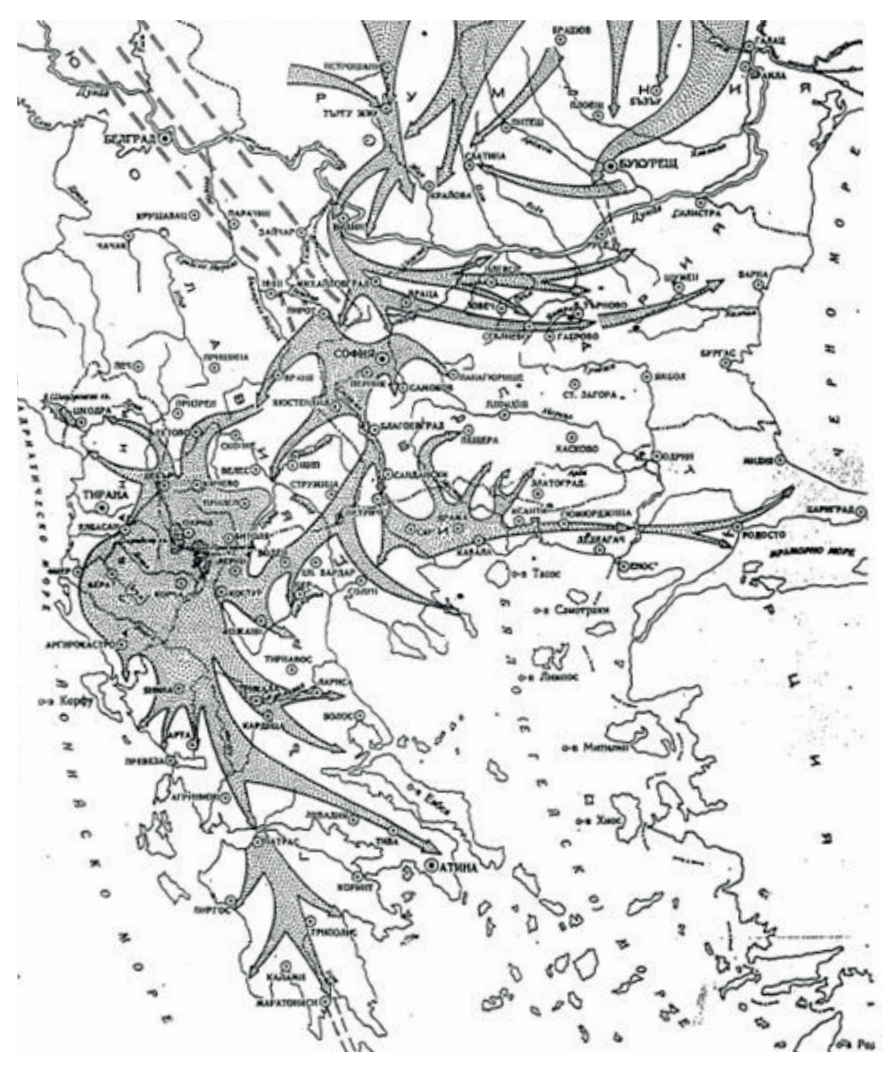

Karte 1. Quelle: Zaimov, 1967, Beilage

Diese Karte von J. Zaimov zeigt allerdings nur die Zuwanderung slavischer Stämme im Osten des Balkans, der Westen ist hier nicht erfasst worden. Diese Lücke kann durch weitere Sammlungen und Kartierungen anderer slavischer geographischer Namen aber geschlossen werden. Ich werde darauf noch zu sprechen kommen.

Hauptzweck meines Beitrages ist es, auf eine sehr umfangreiche Sammlung von geographischen Namen hinzuweisen, die seit einigen Monaten im Internet frei verfügbar ist. Sie entstand im Verlauf der letzten 50 Jahre. Da zu Beginn der 
Sammlung (1970) weder Internet, noch EDV oder Computer zur Verfügung standen, wurde die Sammlung nach der nur damals möglichen Methode angelegt: auf Papier. Eine andere Möglichkeit gab es nicht. Das entsprach damals dem wissenschaftlichen Standard und wurde zum Beispiel bei dem Versuch, einen „Neuen Förstemann“ in Freiburg zu erstellen, angewandt, aber auch für die Sammlung des NMPol in Kraków. Der Autor dieses Beitrages hatte vor einigen Jahren die Möglichkeit, diese Sammlung zu sehen und für einige Recherchen zu nutzen.

Die jetzt neue vorgelegte Sammlung, um die es in diesem Betrag geht, ist nun im Internet frei zugänglich².

Ich gebe im Folgenden eine kurze Beschreibung der Datei und Hinweise zur Benutzung.

\section{ZUR ENTSTEHUNG DER DATEI}

Die Sammlung enthält eine große Menge von geographischen Namen, geschätzt etwa 450.000. Sie war zunächst nur für den slavischen Raum (jetziges und ehemaliges Siedlungsgebiet slavischer Stämme) angelegt. Im Laufe der Zeit wurden dann auch Namen aus angrenzenden Regionen aufgenommen, vor allem Dingen deshalb, weil es sich im Verlauf der Arbeit herausstellte, dass das slavische Siedlungsgebiet Namen enthält, deren Bearbeitung und sinnvolle Deutung nur unter Einbeziehung auch außerslavischer Toponyme und Hydronymie möglich ist.

Die Sammlung ist in erster Linie eine Verweisdatei. Anfangs ist versucht worden, auch Deutungen und Anmerkungen zu den einzelnen Namen hinzuzufügen, aber die Fülle des Materials — das slavische Siedlungsgebiet reicht von der Elbe bis zur Kamtschatka und von der Ostsee bis nach Griechenland - erlaubte schon bald diese Ausweitung nicht. In gewissem Sinn kann die Sammlung mit dem von R. Schützeichel herausgegebenen und von seinen Mitarbeiterinnen und Mitarbeitern bearbeiteten Register der Beiträge zur Namenforschung, Band 1-16, Heidelberg 1969, verglichen werden, ein Register, dass man auch heute noch mit Gewinn nutzen kann.

\section{AUFBAU DER KARTEI}

Kernstück ist die alphabetische Zetteldatei. Sie enthält ca. 450.000 Zettel. Man wird bei der Nutzung sehen, dass es sich um eine relativ einfache Sammlung handelt, man darf sie vielleicht auch als primitiv bezeichnen. Das liegt zu einem Teil daran, dass dem Verfasser zu Beginn der Sammlung nicht bewusst war, wie

${ }^{2}$ Sie steht auf der Internetseite: https://adw-verwaltung.uni-goettingen.de/ortsnamen/images_lightbox.php 
das Verfahren im Einzelnen ablaufen sollte und was sich aus den ersten kleinen Anfängen heraus entwickeln sollte.

Die Hauptkartei ist alphabetisch angeordnet. Einem Namen A a folgen also Aar, Abalon, Achalm usw. Aber es gibt Besonderheiten, die in hohem Maße durch die Alphabete vor allem der osteuropäischen Sprachen und auch die Umsetzung von kyrillischen Schriften bedingt sind. Dazu gehören etwa die Einordnung

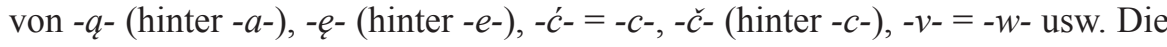
Einzelheiten werden in einem einführenden Abschnitt unter „Entstehungshinweise“ und „Nutzungshinweise“ aufgeführt.

Der Vorgang der Nutzung kann etwa wie folgt umschrieben werden.

Sucht man nach einem bestimmen Namen, z. B. Hubatá, so findet man nach der Suche nach Namen mit dem Anfangsbuchstaben $H$ - und der Unterabteil $H u$ zwischen Hubarna und Hubava die folgende Karteikarte:

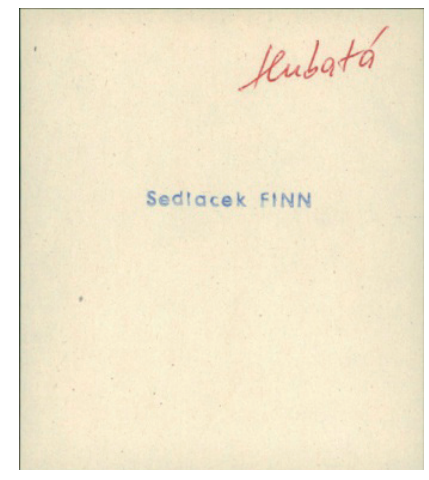

Abbildung 1. Karteikarte Hubatá

Die Karte enthält die Information, dass der Name in einer Publikation ,Sedlacek, FINN" enthalten ist. Im Literaturverzeichnis steht die Auflösung der Abkürzung:

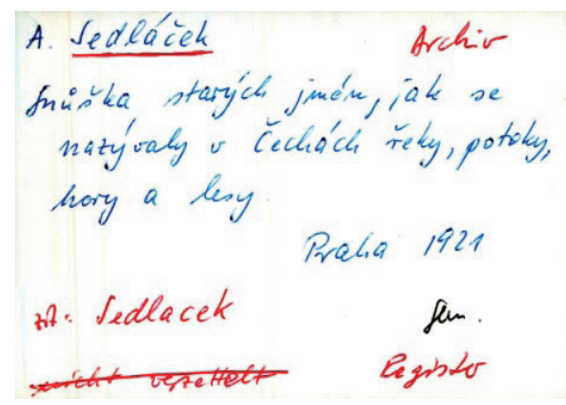

Abbildung 2. Literatureintrag 
Diese Karteikarte enthält auch Information über den Standort der Publikation. Aus der Karte ergibt sich auch, dass die Publikation ein Register enthält, in der weitere Informationen enthalten sind. Darauf verweist auch der Eintrag auf der Karteikarte „Sedlacek, FINN“. Die Publikation enthält nämlich mehrere Register, darunter eines, dass der Autor der Kartei „FINN“ = Flurnamen genannt hat. Greift man zu dem Band von A. Sedláček (1920, heute bequem zugänglich im Internet), so findet man dort das Register Abecední seznam hor a lesův. Darin schließlich steht der Name Hubatá mit der weiteren Angabe „(6-VIII) 71“. Auf der angegebenen Seite 71 steht schließlich der Eintrag „Hubatá něm. Hubata u Domaslavě protí Krasíkovu“. Damit ist der Name lokalisiert und kann kartiert werden.

Man sieht, die Nutzung der Datei ist nicht einfach und die Frage, wo sich ein gesuchter Name befindet und was an der betreffenden Stelle über ihn eventuell gesagt wird, muss dann in der genannten Publikation nachgesehen werden.

\section{BEISPIELE DER NUTZUNG DER KARTEI. WANDERUNG VON SLAVISCHEN STÄMMEN AUF DEN BALKAN}

Ich greife die oben abgebildete Verbreitungskarte südslavischer Stämme von J. Zaimov wieder auf und möchte zeigen, dass eine möglichst umfassende Kartierung von slavischen Orts-, Flur- und Gewässernamen, die bei J. Zaimov erkennbare Zuwanderung slavischer Stämme mit weiteren und sicheren Hinweisen entscheidend stützen kann. Dazu nutze ich die beschriebene Datei.

In den slavischen Sprachen gibt es Appellativa, die in verschiedener Weise von einer Wurzel *mok-, wozu auch močiti 'feucht, nass machen, einweichen, erweichen (z. B. Hanf)' abgeleitet sind, zumeist mit Hilfe des Suffixes *-dlo. Daneben treten aber auch -l-Bildungen auf. Neben močiti gibt es auch einen Verbalstamm močati, der in ähnlicher Weise eine Grundlage bilden kann.

Aus der großen Zahl von Wörtern, die hier genannt werden können, nenne ich nur russ. močalo 'zerfaserter Lindenbast', russ. dial. močilo, močalo 'niedriger, sumpfiger Ort, Wagenspur, Teich', ukrain. mocylo 'kleiner Teich, Pfütze, Ort im Fluß, wo Flachs oder Lein eingeweicht wird', poln. moczadło 'sumpfiger, mit Wasserpflanzen bewachsener Grund, Sumpf', poln., altpoln. (seit dem 13. Jahrhundert) moczydło „,nasser Boden, Sumpfboden, Sumpf, Morast, Moor, Lache, Tümpel', tschech., slovak. močal, močalina, močalisko u.a. 'Sumpf, Morast, Moor, feuchte Stelle auf einem Feld oder einer Wiese'.

Auch im Slovenischen, Sorbischen, Kroatischen und Serbischen sind Entsprechungen bezeugt. Aus dem Slavischen entlehnt sind alban. moçali, maçal 'Morast' und ungar. mocsolya 'Pfütze, Hanfröste'.

Soweit ein kurzer Blick in die Appellativa. Wichtiger aber ist die Frage, wo sich davon abgeleitete geographische Namen finden, wie sie gestreut sind und wo sie fehlen. Dazu trägt die angesprochene Datei entscheidend bei. 
Sie enthält — geschätzt — ca. 200-300 Orts-, Flur- und Gewässernamen. Im Einzelnen sind die folgenden Ableitungen:

Moczydto, Močidlo, Močidto, Moczadło, Močil, Mociul, Močul', Moczel, Močidla, Močidła, Modschiedl, Moczydła, Močila, Močilla, Močily, Močidly,

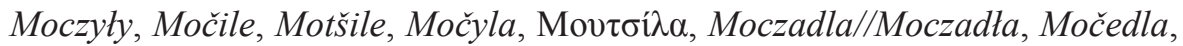
Močály, Moczadty, Maczuly, Mačuly, Maczule, Močuly usw. Es gibt noch weitere Varianten, z. B. durch deutschen, rumänischen und ungarischen Einfluss lautlich umgestaltet.

Ebenfalls häufig sind, wie im Slavischen nichts anders zu erwarten, Ableitungen mit Suffixen. Die Datei enthält z. B. das im Slavischen häufige Suffix -ica in Mötzlich, Ort bei Halle, Močidlice, Moczalica. Häufiger sind Erweiterungen mit *-(b)- wie Močilnica, Moczydlnica. Ein Suffix *-bc- liegt vor in Močidlec, Močilec, Moczulec, Mačýlca, Močidlce. Das Bildungselement *isk(ý)o- darf vermutet werden in Mačališče, Močalište, Mačulišče, Močylyšča u.a.m.

Schließlich erwähne ich hier noch Bildungen mit dem Suffix *-(b)n-ica: Močilnica, Moczydlnica. Weitere Bildungen sind Namen mit den Suffixen *-bc-,

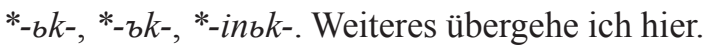

Diese stereotype und vielleicht als eintönig empfundene Sammlung gewinnt ihren Wert durch deren Kartierung (Karte 2).

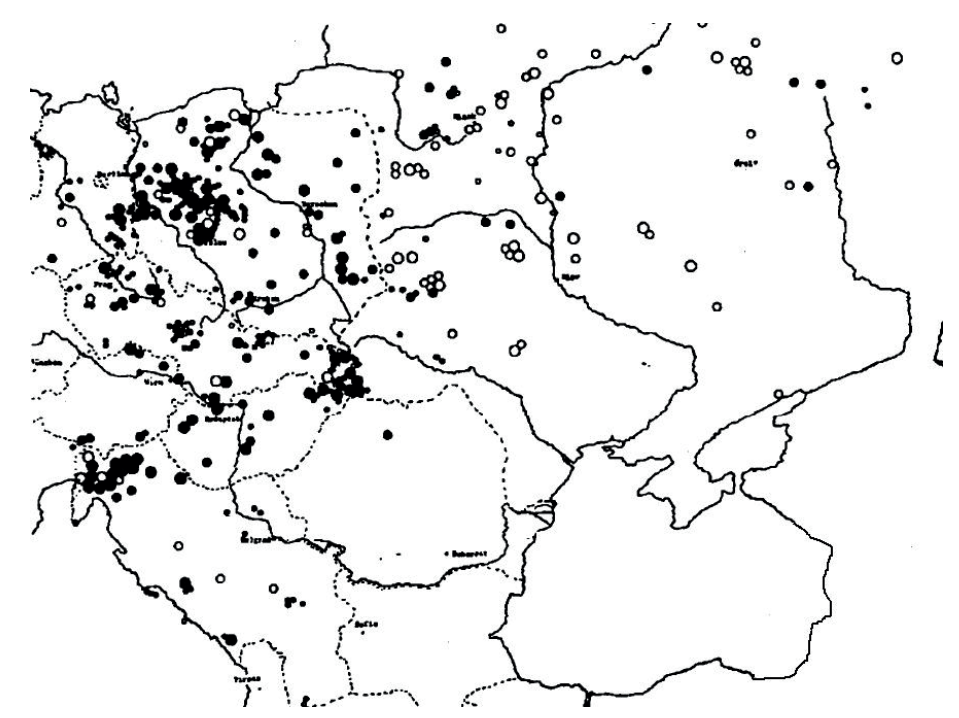

Karte 2. Verbreitung von močylo, moczydto in slavischen Ortsnamen

Der Schwerpunkt dieser geographischen Namen liegt im westslavischen Bereich, vor allem Polen hat daran einen hohen Anteil. Daneben fallen 
Konzentrationen im slovakisch-ukrainischen Grenzgebiet und in Slovenien auf. Vor allem aber fällt auf, dass das Ostslavische nur geringen Anteil an der Verbreitung hat.

Ich sehe hier die Möglichkeit zu folgender Interpretation: wir wissen, dass slavische Stämme etwa seit dem 5./6. Jh. auf ihrer Ausbreitung nach Süden aus einem Bereich nördlich der Karpaten gekommen sein müssen. Ich denke, es ist nicht zu gewagt, wenn man die Ausbreitung in Ungarn und Slovenien als Kennzeichen der Siedlungsbewegung von dem Bereich nördlich der Karpaten auf den Balkan interpretiert. Dabei bietet sich als ein Bereich der Wanderung seit der Bronzezeit, aber wahrscheinlich auch schon früher genutzte Weg durch die Mährische Pforte an. Hier wird auch zu mindestens ein Zweig der Bernsteinstraße vermutet, die schon in der Tabula Peuteringiana beschrieben worden ist.

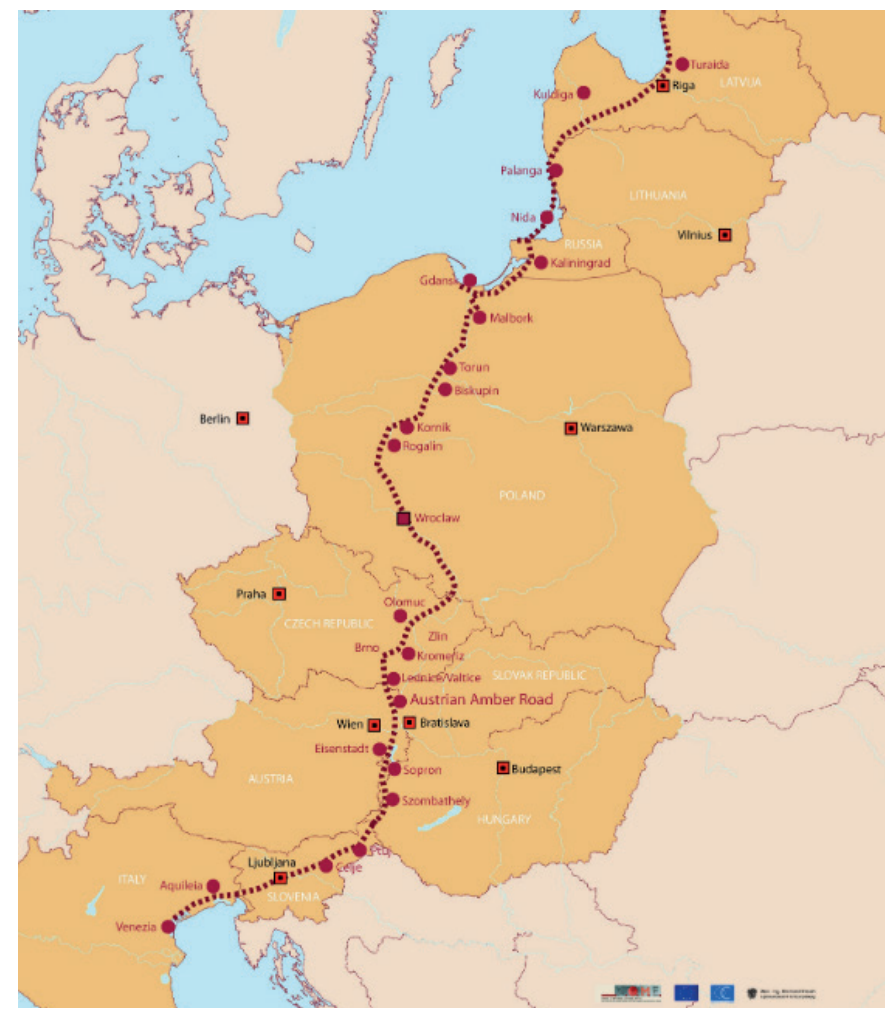

Abbildung 3. Bernsteinstraße

Von Slovenien aus greifen dann einzelne Spuren der slavischen Namen über Kroatien nach Serbien, Albanien und Nordmazedonien aus. Dann enden die Ortsnamen. 
Auffallend ist, dass Rumänien und Bulgarien nur geringe oder gar keine Ortsnamen kennen.

Bevor ich weitere Schlüsse ziehe, empfiehlt es sich, anhand der Datei ein weiteres Appellativum in gleicher Weise zu untersuchen.

Ich habe dazu slavisch luža ausgewählt. Man findet es als ein auch heute noch bekanntes Wort im Ukrainischen (lúža, lužok), im Weißrussischen (lúža, auch als lúžyna usw.), schon im Altrussischen (luža), in polnischen Dialekten (luża, auch als tužyna), im Sorbischen (luža), im Tschechischen und Slovakischen (louže, louž, luža), alttschechisch lúže, ferner im Slovenischen (luža, auch als lúžica) und im Kroatischen und Serbischen als luža . Es bedeutet u.a. „Pfütze, Lache, mit Wasser gefüllte Vertiefung, Sumpf, Teich, Morast“ u.a.

Es wird übereinstimmend auf indogermanische Grundlagen zurückgeführt und es ist als Lusche „unreine Flüssigkeit, Teich, Flüssigkeitsansammlung“ in das Deutsche entlehnt worden.

In geographischen Namen kann es recht sicher nachgewiesen werden. Aus der erwähnten Datei nenne ich hier nur in Auszügen:

Luž, auch Lausche, Łuža, Luža, Louž, Louže, Luže, Łuże, ferner in zumeist eingedeutschter Form als Laus, Laussa, Lausche, Lause, Lauße, Luscha, Lusche, Lauz und Lusua belegt. Mit Suffixen sind gebildet Lužac, Łużec, Lužec. Bei Namen wie Lužná, Loužná und ähnlichen nicht sicher, ob sie von *luža abgeleitet sind oder von *log-. Bei Ableitungen mit *-bk-//*-ъk- liegt zumeist luža mit seinen Entsprechungen vor: Loužek, Łużek, aber letzte Sicherheit kann man nicht gewinnen. Auf weitere, zumeist unsichere Namen gehe ich hier jetzt nicht näher ein.

Wichtig ist nun die Kartierung (Karte 3).

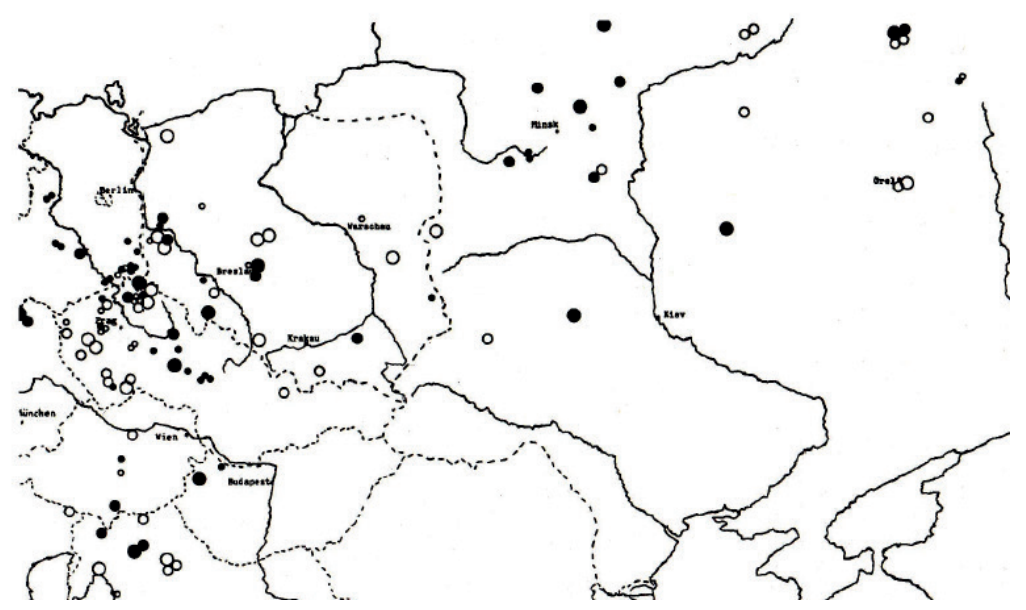

Karte 3. Verbreitung von *luža in slavischen Ortsnamen 
Die Verbreitung lässt Häufungen im russischen und weißrussischen, im böhmisch-altsorbischen und im slovenischen Sprachgebiet erkennen. Es gibt Deckungen mit der Streuung der von močylo bzw. moczydto abgeleiteten Namen und es zeigt sich deutlich, dass die Namen zum Balkan hin den Westen in Slovenien und ein wenig noch in Kroatien erreichen, den Osten aber bis hin zu Bulgarien nicht berühren. Wir werden gleich an anderen Verbreitungen slavischer Namen sehen, dass es auch ganz andere Verbreitungen gibt, die den Westen (Slovenien, Kroatien, Albanien) völlig aussparen und entgegengesetzt im Osten (Moldau, Dobrudscha, Bulgarien) ihr Verbreitungsgebiet haben.

Es gibt weitere slavische Wasserwörter, deren Vorkommen in geographischen Namen die Zuwanderung von slavischen Stämmen nach Süden auch für den Osten des Balkans deutlich nachzeichnet. Es geht hier um eine Bewegung, die von den alten Siedlungsgebieten nördlich der Karpaten dem Lauf der Gebirgskette folgt und über Moldau und die Dobrudscha Bulgarien erreicht und z.T. noch weiter nach Süden vorstößt. Dazu biete ich im Folgenden zwei Beispiele.

Zum einen sind es Ortsnamen, die von slavisch izvor "Quelle" abgeleitet sind, zum andern ist slavisch bagno „Sumpf, Morast“ das zugrunde liegende Wort.

Ein slavisches Appellativum, das vor allem in der Bedeutung „Quelle“ in den slavischen Sprachen bezeugt ist, lässt sich am ehesten auf einen urslavischen Ansatz *jbz-vorz zurückführen. Hierzu gehören u.a. schon altrussisch izvorb „Quelle“, ukrain. jezviro, izvór, zvir, izvir, -oru u.a.m. „Niederung zwischen Erhebungen; Quelle; Schlucht; Schlucht, in der ein Bach fließt" u.ä., poln. zwór, -oru, zwora, -y (mit etwas abweichender Bedeutung) ,Rinne zwischen zwei Bergen in den Karpaten“, bulgar. izvor „Ort, wo Wasser herausfließt“, serb., kroat. izvor, zvir, izvir „Quelle, Born, Strudel“, altserb. izvorb „,fons“, sloven. izvòr, -vóra „Quelle, Ursprung, Grund“, izvornik „Quelle“, altkirchenslav. izvorz „Quelle“.

Das slavische Wort ist in das Rumänische entlehnt als izvór, plural. izvoáre, -voára „Quell, Quelle“, ins Aromunische als izvur, ins Albanische als xvor, dzvor,

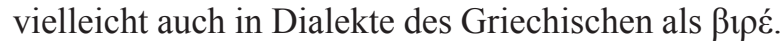

Die Zuordnung von geographischen Namen zu den slavischen Wörtern ist zumeist unkompliziert. Die angesprochene Datei bietet eine Fülle von Beispielen: Izvor, Izvur, Izwór, Izwor, Izvór, Izvóry, Iswor, İzvar. Mit westslavischer und ukrainischer dialektaler Lautentwicklung *jbz- $>z$ - kann man hier anschließen: Zwor, Zwór, Źwir, Zwór, Zvür und andere. Auch Formen mit Artikel sind belegt: Izvorŭt, Ízvarŭt, İzvurŭt, İ̀voreto, Izvorjeto, Ízvorete, İzvurti, ebenso wie Pluralformen: Izvori, Izvory, Izwory, Zwori, Zwory u.a.m.

Weiter gibt es Namen, die offenbar von Rumänen gegeben worden sind. Ich habe sie dennoch aufgenommen, weil deren geographische Streuung wichtig für die Bestimmung möglicher Kontaktzonen zwischen Slaven und Rumänen sein dürften: Izvoare, Izvora, Izvore, Izvoáre, Izvoarele, Izvoralu. Auch Suffixbildungen 
fehlen nicht, ich nenne hier nur: Izvorec, Zworzec, Zworec, Zvoreč, Zworzecz, Izvorci, Izvorštica, Izvoršnica, Zvoryk, Zvirnyk, Zvornik, Izvorsko, Izvorište, Izvorovo, Ivzórak, Zvórja u.a.m.

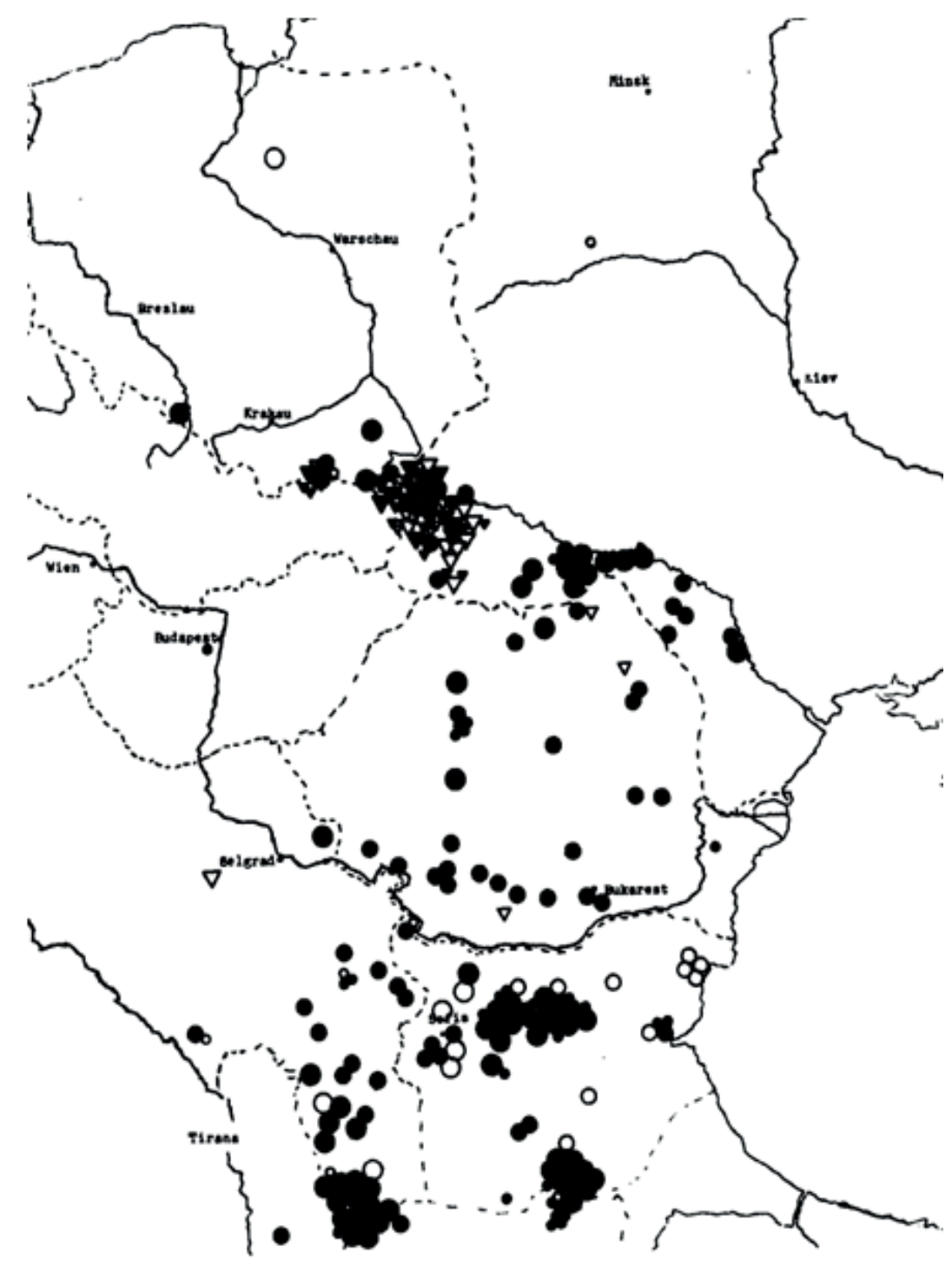

Karte 4. Verbreitung von $*_{j b z v o r b}$ in slavischen Ortsnamen 
Mit einem Blick erkennt man, dass die Verbreitung dieser Namen entscheidend von der der vorigen Karten, also der Verbreitung von luža und močylo, moczydło abweicht: Ungarn, Slovenien, Kroatien und Albanien haben keinerlei Anteil an der Streuung. Diese reicht vielmehr ausgehend von ihrem Zentrum nördlich der Karpaten über die Bukowina bzw. Siebenbürgen/Rumänien bis nach Serbien, Bulgarien und Nordgriechenland. Es ist ein ganz anderer Verbreitungsweg als bei den oben behandelten zwei Appellativen. Das kann noch durch ein weiteres „Wasserwort" und dessen Vorkommen in geographischen Namen verdeutlicht und verstärkt werden.

Es geht um slavisch bagno, ein Wort, das in den slavischen Sprachen wie folgt bezeugt ist: russ. dial. (nur in den an die Ukraine und Weißrußland angrenzenden Gebieten) bagnó „niedrige, sumpfige Stelle, Morast“", ukrain. báhno „Sumpf, Morast" (dazu Ableitungen wie bahnýśko, bahnýšče, bahnovys 'ko mit ähnlichen Bedeutungen), weißruss. báhna ,unzugänglicher Torfsumpf, Sumpf, Morast“, poln. bagno „Sumpf, Moor“ (mit Ableitungen wie bagnowisko), schon altpoln. bagno „palus, stagnum, limus“, auch „ledum palustre“", sorb. bahno, bagno „Sumpf, Morast, Bruch; Sumpfporst“" (dazu Ableitungen wie bahniško, bahnišćo, bahnowizna u.a.), polab. bógnö „Sumpf“, tschech. bahno „Sumpf, Schlamm, Morast“, dial. auch behno (mit Ableitungen wie bahnisko, bahniště, bahnovina u.a.), slovak. bahno „Sumpf, Morast“. Im Südslavischen sind Entsprechungen nicht nachzuweisen, aber das Wort ist entlehnt in das Rumänische als bahnă.

Der Blick in die Namen zeigt starkes Vorkommen in der Lausitz, in Nord-, West- und Südpolen und im ukrainischen Vorkarpatenland. Die folgenden Namen lassen sich mit Hilfe der hier in Rede stehenden großen Namendatei nachweisen und kartieren:

Bagno (sehr häufig), Bahno, Bahne, Begno, plurale Bildungen wie Bagna, Báhna, Bahna, Behna, Bahnă, Suffixbildungen wie Bahneć, Bahenec, Bagenz, Bagnowce, Bagieniec, Bahnica, Bahniţa, Bagnica, Bagienica, Bagienice, Bagiennica, Bagienek, Bagienka, Bagienko, Bagienki, Bagieneczko, Bagnówka, Bahnívka, Bagniewko, Bagniewka, Bagniska u.a.m.

Ein eindrucksvolles Bild bietet die Verbreitung der Namen (Karte 5).

Obwohl das Appellativum vor allem im westslavischen Bereich sehr gut und stark verbreitet ist, streuen die Namen nicht in Richtung Österreich und Slovenien und weiter nach Süden aus. Es ist ein ganz anderer Weg erkennbar, der besonders deutlich wird, wenn man den Verlauf der Karpaten zum Vergleich hinzunimmt (Karte 6).

Es ist ganz klar, dass die Verbreitung der von bagno abgeleiteten Namen eine Ausbreitung nach Süden entlang dem Karpatenbogen markieren. Da es um ein slavisches Wort geht, ist es nicht allzu gewagt anzunehmen, dass es sich um eine Siedlungsbewegung handelt, die durch Sprecher von slavischen Dialekten getragen worden ist. 


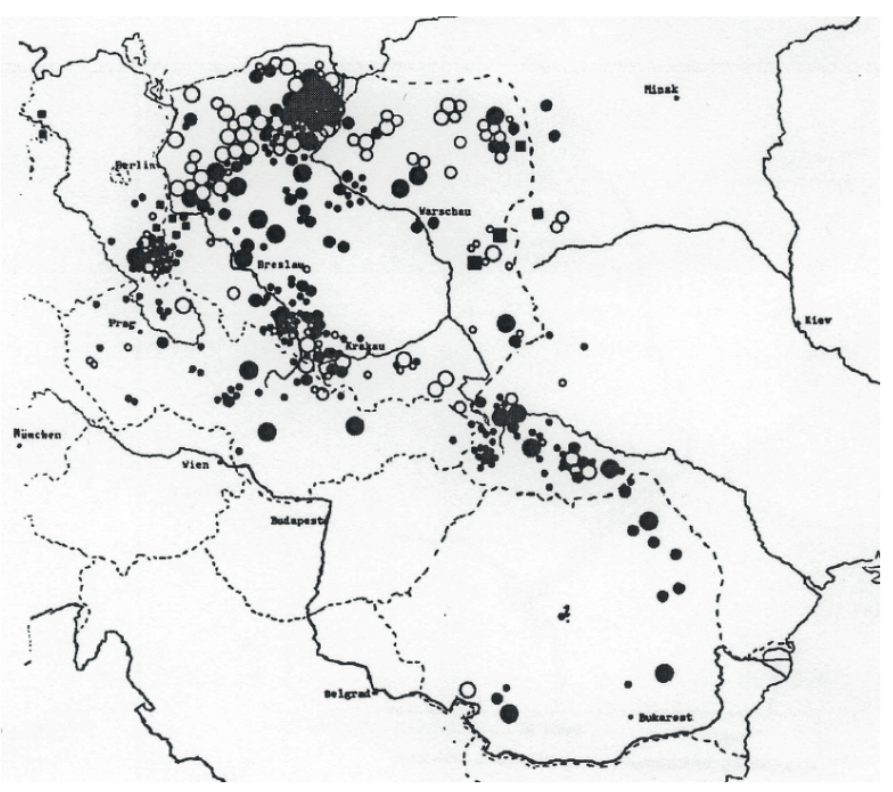

Karte 5. Verbreitung von *bagъno in slavischen Ortsnamen

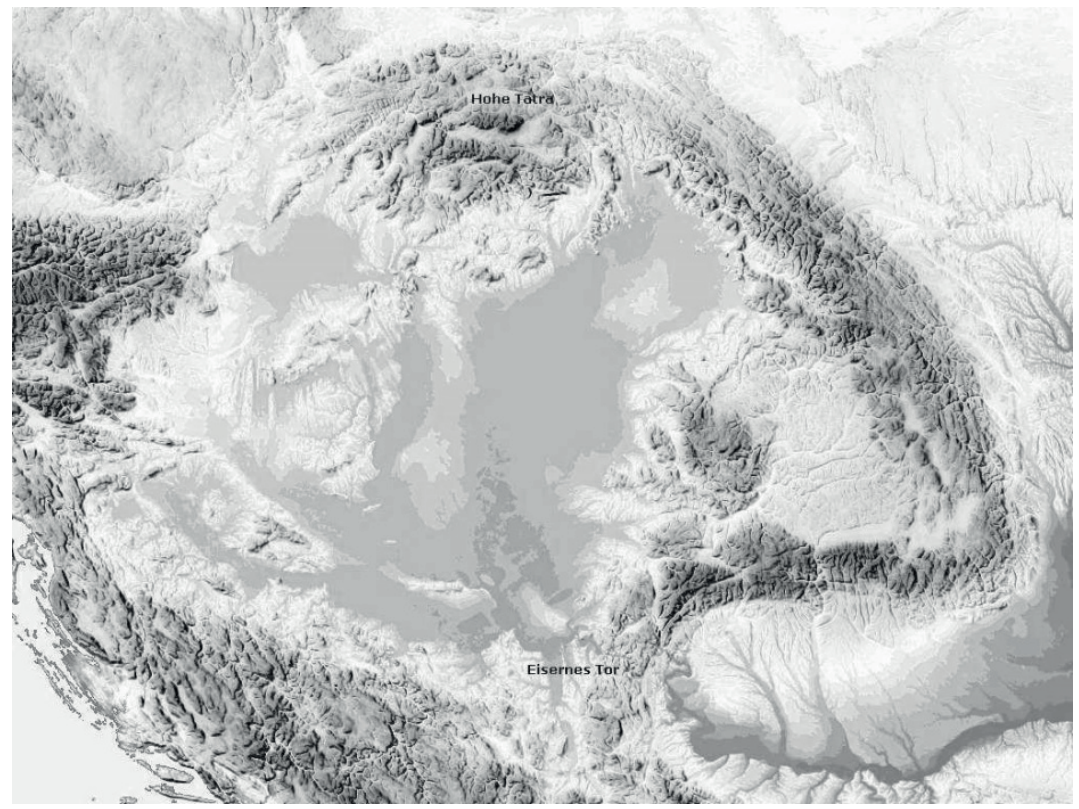

Karte 6. Verlauf der Karpaten 
Anhand dieser vier behandelten slavischen Appellativa, die durch weitere Beispiele ergänzt werden können, lässt sich zeigen, dass der Balkan durch Slaven auf unterschiedlichen Wegen erreicht worden ist: zum einen durch einen Zuzug aus der Mährischen Pforte heraus über den Osten Österreichs und Ungarn hinweg nach Slovenien und Kroatien, und weiter nach Albanien und den Westen Serbiens bis nach Westgriechenland. Der andere Zuzug erfolgte entlang dem Karpatenbogen durch die Bukowina, Moldau, die Dobrudscha (teilweise durch Rumänien/Siebenbürgen) und die untere Donau bis hin nach Bulgarien und den Osten Griechenlands.

Es geht mir hier also vor allem um die Ausbreitung nach Süden, zum Balkan hin. Die unterschiedlichen Zuwanderungswege haben nämlich auch im Wortschatz des Rumänischen und des Serbischen ihre Spuren hinterlassen. Das möchte ich jetzt erörtern.

Es lässt sich ermitteln, dass eine Entlehnung in das Rumänische nur dann erfolgte, wenn die Wanderung entlang der Karpaten nach Südosten erfolgte: Slavische Wörter, die die Grundlage für entsprechende Ortsnamen in Rumänien, in Moldau und der Dobrudscha bilden, sind in das Rumänische entlehnt worden:

\begin{tabular}{|c|c|}
\hline slavisch & rumänisch \\
\hline *jbzvorb & izvór \\
\hline bagno & bahnă \\
\hline sigla & sihlă, sîhla \\
\hline stubel & 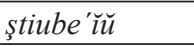 \\
\hline
\end{tabular}

Die unterschiedlichen Wege der Einwanderung haben auch im Wortschatz der südslavischen Sprachen ihre Spuren hinterlassen. Ausführlich hat sich damit Popović (1960, S. 438-444) beschäftigt. Aus seinen Kartierungen des Wortschatzes des Südslavischen habe ich drei Karten ausgewählt.

Zunächst ein Wort für „Regen“ (Karte 7):

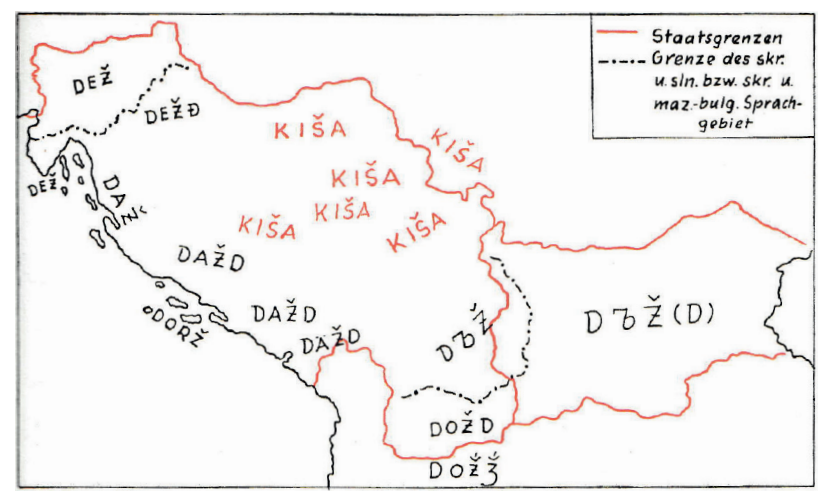

Karte 7. „Regen“ in den südslavischen Sprachen. Quelle: Popović, 1960, S. 441 
Serbisch und kroatisch $k i s ̌ a$ ist zweifellos das jüngere Wort gegenüber Fortsetzern von *dzždb „Regen“, vgl. poln. deszcz, russ. dožd' usw.

Ganz ähnlich ist die Verbreitung bei dem Wort für „Eisen“ (Karte 8):

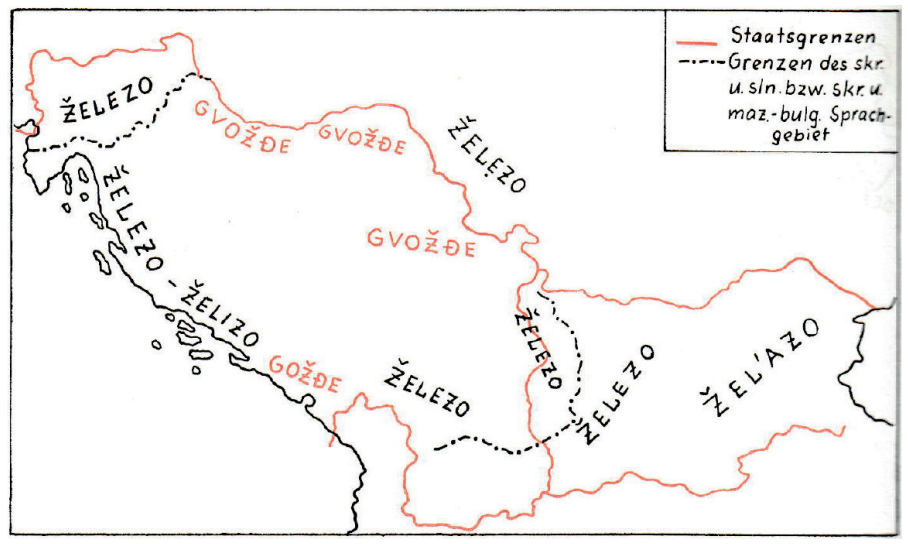

Karte 8 „Eisen“ in den südslavischen Sprachen. Quelle: Popović, 1960, S. 442

Das jüngere Wort ist serbisch und kroatisch gvožđe, gožđe, das ältere das zweifellos aus einer vorslavischen, indogermanischen Sprachschicht stammende *želězo, man denke an russ. železo, poln. żelazo. Und ein letztes Beispiel: das slawische Wort für „Ruß“ (Karte 9):

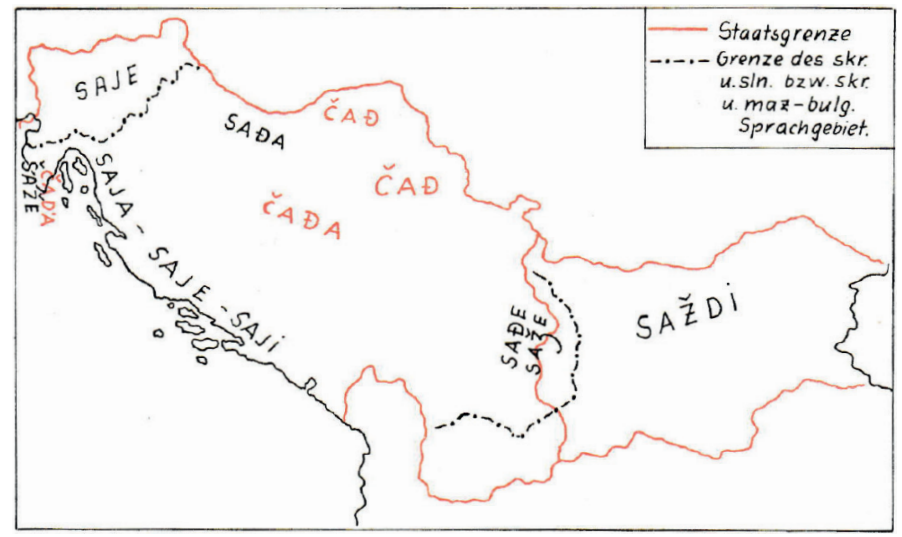

Karte 9. „Ruß“ in den slavischen Sprachen. Quelle: Popović, 1960, S. 443

Das ältere Wort ist zweifellos das mit lit. súodžiai und altenglisch bzw. altnordisch sót verwandte „Ruß“-Wort *sad'a. 
Man darf daraus folgern, dass die Erhaltung der Archaismen an der Peripherie des südslavischen Sprachgebietes mit der Zuwanderung aus den alten Wohnsitzen der Slaven nördlich der Karpaten zusammenhängt. In der Mitte, in der sich die beiden Siedlungsströme dann wieder trafen, entstanden Neuerungen oder es setzten sich andere slavische Wörter wie etwa *sad'a durch.

Die Zuwanderung slavischer Stämme nach Süden darf nach Ausweis der geographischen Namen, die auf slavischen Bezeichnungen für ,Wasser, Fluss, Bach, Morast, Sumpf, Teich usw.“ basieren, etwa wie folgt umrissen werden (Karte 10).

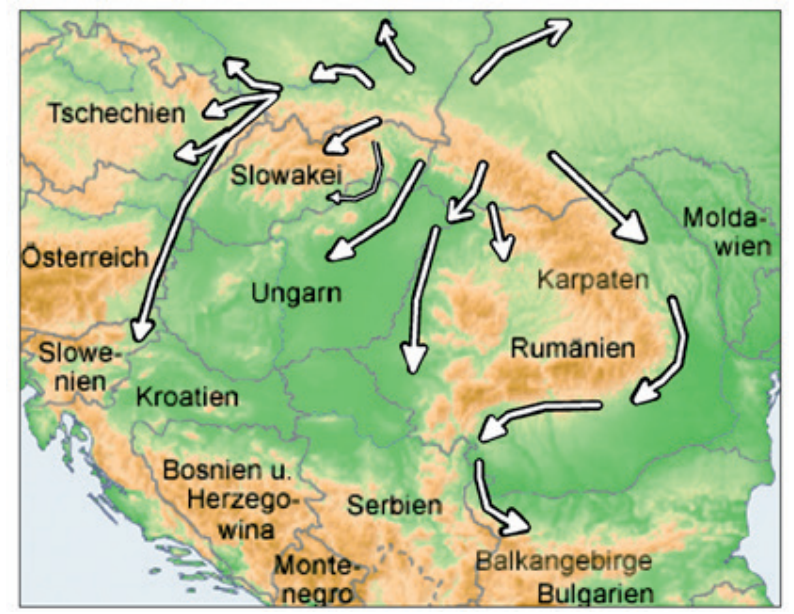

Karte 10. Ortsnamen und Ausbreitung slavischer Stämme

Ich erinnere an dieser Stelle an die oben abgedruckte Karte 1 von J. Zaimov (1967), auf der die Zuwanderung slavischer Stämme im Süden des Balkans umrissen wird. Die durch die Sammlung slavischer Namen in der angesprochenen Internetdatei möglich gemachten Kartierungen von ausgewählten slavischen „Wasserwörtern“ bilden sozusagen den nördlichen Teil des Balkans ab und umreißen die beiden großen Wanderungswege slavischer Stämme: Österreich, Ungarn, Slovenien und Kroatien auf der einen Seite, die Bukowina, Moldau, Dobrudscha, das untere Donaugebiet und Bulgarien auf der anderen Seite.

Es war die Absicht dieses Beitrages, anhand ausgewählter Sammlungen und Kartierungen auf die Möglichkeiten, die sich durch die hohe Zahl der gesammelten Namen in der angesprochenen Datei $^{3}$ für die Bearbeitung slavischer Namen ergeben können, hinzuweisen.

Ich beschließe diesen Beitrag zu Ehren von Barbara Czopek-Kopciuch mit dem Hinweis darauf, dass Material in der Internetsammlung auch durch Aufenthalte

\footnotetext{
${ }^{3}$ https://adw-verwaltung.uni-goettingen.de/ortsnamen/images_lightbox.php
} 
in der Arbeitsstelle des Instituts für Polnische Sprache der Polnischen Akademie der Wissenschaften ermittelt werden konnte und in die Sammlung eingeflossen ist. Barbara Czopek-Kopciuchs Hinweise waren dabei eine liebenswürdige und wertvolle Hilfe, an die ich noch immer zurückdenke. Das betrifft auch spätere Begegnungen und Kontakte. Wie sicherlich viele in der onomastischen Welt werde ich den Kontakt mit ihr vermissen.

\section{LITERATUR}

Eiler, W. (1982). Geographische Namengebung in und um Iran. Ein Überblick in Beispielen. München: Verlag der Bayerischen Akademie der Wissenschaften.

Fring s, Th. (1957). Grundlegung einer Geschichte der deutschen Sprache (3. Aufl.). Halle (Saale): Niemeyer.

NMPol = Rymut, K., Czopek-Kopciuch, B., \& Bijak, U. (Hrsg.). (1996-). Nazwy miejscowe Polski. Historia. Pochodzenie. Zmiany [Place Names of Poland. History. Origin. Changes] (Bd. 1 ff.). Kraków: Instytut Języka Polskiego PAN.

Popović, I. (1960). Geschichte der serbokroatischen Sprache. Wiesbaden: Otto Harrassowitz. Sedláček, A. (1920). Snůška starých jmen, jak se nazývaly v Čechách řeky, potoky, hory a lesy. Praha: Rozpravy České akademie věd a umění. https://www.academia.edu/16258149/August_ Sedláček_Snůška_starých_jmen_jak_se_nazývali_v_Čechách_řeky_potoky_hory_a_lesy_ August_Sedláček_a mountains and forests

SSNO = Taszycki, W. (Hrsg.). (1965-1984). Stownik staropolskich nazw osobowych [Dictionary of Old Polish Personal Names] (Bd. 1-7). Wrocław: Zakład Narodowy im. Ossolińskich.

Udolph, J. (1979). Studien zu slavischen Gewässernamen und Gewässerbezeichnungen. Ein Beitrag zur Frage nach der Urheimat der Slaven. Heidelberg: Carl Winter Universitätsverlag.

Zaimov, J. (1967). Zaselvane na bălgarskite slavâni na Balkanskiâ poluostrov [Settlement of Bulgarian Slavs on the Balkan Peninsula]. Sofija: Bălgarska Akademiâ na Naukite.

\section{SUMMARY}

A NEW BIBLIOGRAPHICAL COLLECTION OF EUROPEAN PLACE, FIELD AND WATER NAMES INSTRUCTIONS FOR USE

The article discusses a file with around 450,000 geographical names that has been freely accessible on the Internet for a few months. The title of this file is Nomina Geographica Europaea. Bibliographische Sammlung zu europäischen Orts-, Flur- und Gewässernamen reachable at the following Internet address: https://adw-verwaltung.uni-goettingen.de/ortsnames/images_lightbox.php/.

The text describes the file in more detail. Its origin, principles and structure are outlined and reference is made to the fact that its use is completely free and open to anyone interested.

In the second part of the article, an example of the migration of Slavic tribes to the Balkans is introduced to show how the file with its large amount of data can be used: the collection of geographical names allows, thanks to the information it contains, to create mappings, the results of which are important for the question of the routes by which Slavic tribes made their way south.

Keywords: place names, field names, water names, Europe, bibliography 